\title{
Process support for risk mitigation: a case study of variability and resilience in vascular surgery
}

\author{
Berit Brattheim, ${ }^{1,2}$ Arild Faxvaag, ${ }^{2}$ Andreas Seim ${ }^{3,4}$
}

\begin{abstract}
${ }^{1}$ Department of Radiography, Sør-Trøndelag University College (HiST), Trondheim, Norway

${ }^{2}$ Norwegian EHR Research Centre (NSEP), Department of Neuroscience, Faculty of Medicine, Norwegian University of Science and Technology (NTNU), Trondheim, Norway ${ }^{3}$ Department of Computer and Information Science, Norwegian University of Science and Technology (NTNU), Trondheim, Norway ${ }^{4}$ SINTEF Technology and Society, Industrial Management,Trondheim, Norway
\end{abstract}

Correspondence to Berit Brattheim, Department of Radiography, Sør-Trøndelag University College (HiST), MTFS, 0 Kyrresegt 9, N0-7489 Trondheim, Norway; berit.j.brattheim@ntnu.no

Accepted 19 January 2011 Published Online First 16 February 2011

\section{ABSTRACT}

Objective: To inform the design of IT support, the authors explored the characteristics and sources of process variability in a surgical care process that transcends multiple institutions and professional boundaries.

Setting: A case study of the care process in the Abdominal Aortic Aneurysm surveillance programme of three hospitals in Norway.

Design: Observational study of encounters between patients and surgeons accompanied by semistructured interviews of patients and key health personnel. Results: Four process variety dimensions were identified. The captured process variations were further classified into intended and unintended variations according to the cause of the variations. Our main findings, however, suggest that the care process is best understood as systematised analysis and mitigation of risk. Even if major variations accommodated for the flexibility needed to achieve particular clinical aims and/or to satisfy patient preferences, other variations reflected healthcare actors' responses to risks arising from a lack of resilience in the existing system. On this basis, the authors outlined suggestions for a resilience-based approach by including awareness in workflow as well as feedback loops for adaptive learning. The authors suggest that IT process support should be designed to prevent process breakdowns with patient dropouts as well as to sustain risk-mitigating performance.

Conclusion: Process variation was in part induced by systemised risk mitigation. IT-based process support for monitoring processes such as that studied here should aim to ensure resilience and further mitigate risk to enhance patient safety.

\section{INTRODUCTION}

Developing IT-based process support is seen as a promising avenue towards improving efficiency and quality in healthcare. ${ }^{1}$ Such computer-based support may facilitate the flow of patients and information, but also enable the monitoring of tasks and data during care processes to ensure adherence to best practice and clinical guidelines. ${ }^{1-4} \mathrm{~A}$ key design decision in the development of such systems is to the extent to which one should use the system to manage or to reduce process variation.

Modern theory on quality postulates that quality is inversely proportional to variation, ${ }^{5}$ which can be seen as a barrier to performance and quality. ${ }^{6-8}$ Variation is often substantial in healthcare processes and is often reduced by managing variations falling outside specified limits. ${ }^{8}{ }^{9}$ However, process variation may also be an expression of flexibility ${ }^{10}$ reflecting the problem-solving nature of clinical work. ${ }^{11} 12$ Resilience engineering theory suggests that process variation related to flexibility is an integral part of how actors deal with uncertainty, variability and high risk, enhancing safety in unpredictable settings. ${ }^{13} 14$ The resilience engineering approach to managing variations centres on attention to essential properties of adaptive behaviours. ${ }^{15}{ }^{16}$ Hollnagel proposes four properties that he characterises as cornerstones of resilience: (a) the ability to anticipate forthcoming events, (b) attentivity to critical issues, (c) the ability to respond effectively to (unexpected) events, and (d) a willingness and readiness to gain knowledge by learning from both positive and negative experiences. ${ }^{17}$

Given these perspectives on process variations, we conducted a field study of existing work practices in a care process for patients suffering from a pathological widening of their main aorta-abdominal aortic aneurysm (AAA) - across one university hospital and two community hospitals owned by one of the four Norwegian Regional Health Authorities. Our purpose was to identify and describe the characteristics of process variability within and between the hospitals and 
to explore types and sources of variations in the studied process. Based on this study, we develop suggestions for how IT-based process support could be designed to enhance resilience in this clinical process, that is risk mitigation.

\section{Clinical case}

A patient's aneurysm may remain asymptomatic for years before progressively enlarging. The risk of aneurysm rupture is a major concern owing to a high overall mortality. ${ }^{18}$ Thus, AAA patients need to be monitored regularly until their condition reaches the surgical intervention threshold. Two treatment alternatives exist: conventional open surgery or endovascular repair (EVAR) ${ }^{19}$ AAA patients' suitability for EVAR depends on anatomical features of the aorta, limiting use of this treatment alternative. However, the EVAR technique continuously develops and improves, increasing the number of patients included over time. ${ }^{20} 21$

\section{METHOD}

We studied a care process in the AAA surveillance programme of one university hospital and two community hospitals between December 2006 and May 2007. We conducted an observational study and semistructured interviews of patients and key personnel at the surgical outpatient clinic and the radiological department of the three hospitals. All three hospitals offered both the AAA surveillance programme and open-surgery service, whereas the university hospital also performed EVAR.

The vascular surgery service of the university hospital was staffed with five or six surgeons, who in the case of EVAR cooperated with four or five interventional radiologists at the radiological department. Each of the two community hospitals employed one vascular surgeon, also responsible for referring eligible EVAR candidates to the university hospital. ${ }^{22}$ The community-hospital radiologists were not directly involved in EVAR procedures, but some were familiar with EVAR and partly involved in referring patients to further EVAR assessment. Each of the surgical units held a nurse role to facilitate patient care.

Observations focused on one episode of monitoring for AAA patients potentially eligible for vascular surgery. Patients suffering from dementia were excluded from the study. The observations included preparations for the encounter, the patient-surgeon encounter itself (lasting 4-30 min), as well as postencounter tasks. In total, we observed 29 patients (22 males, seven females) with an AAA diameter ranging from 4.1 to $6.5 \mathrm{~cm}$, and with age ranging from 57 to 94 years. We applied a semistructured observation protocol and recorded patient characteristics, aneurysm-related parameters, actors, roles, information sources, events (communicative acts, examination and other events) and statements from the participants. Observations were accompanied by semistructured patient interviews (lasting 15-35 min).

We conducted semistructured interviews with $15 \mathrm{key}$ informants. At the university hospital, this included one nurse/coordinator, three vascular surgeons and three interventional radiologists. At each community hospital, we included two experienced radiologists, one vascular surgeon and one nurse. The interview guide was influenced by the prior observational study. ${ }^{22}$ Each interview lasted 45-60 min.

Data analysis involved three steps:

1. Observations: selected situations were abstracted into scenarios describing work practice pattern.

2. Interviews: we conducted a content analysis of interview transcriptions (applying QSR NVIVO 7) to expand the scenarios and descriptions of work practice patterns.

3. Guided by references, ${ }^{1023}$ we identified and described dimensions, types and sources of process variability.

\section{RESULTS}

Figure 1 is a flow-chart illustration of the principal workpractice pattern and decision points of the AAA monitoring process, describing main activities and actions as well as their principal sequence. The bottom right-hand corner of figure 1 illustrates some of the observed variations of work sequences. Box 1 provides a more detailed description of the monitoring process and also shows some of the observed process variations. We found substantial process variability pertaining to the length of monitoring intervals, the choice of imaging modality, the order of activities (deviations from the order depicted in figure 1) and flow of information. These variations could be categorised as follows:

- sequence: variety in the order in which actions and interdependent activities were performed;

- activity: variety in the number of different activities that constituted the enactment of a particular part of the monitoring trajectory-for example, adding an extra imaging exam or lab test in connection to a monitoring episode;

- place: activities were carried out at different geographical locations and by different organisational units-for example, having an imaging analysis or cardiopulmonary tests at the community hospital while consulting with the surgeon at the university hospital;

- time: variety in (a) the duration of an activity (eg, the radiological report turnaround time differed between units); and (b) the length of time between activities (eg, monitoring intervals). 
Figure 1 Flow chart of principal abdominal aortic aneurysm (AAA) monitoring trajectory. The enclosed box indicates the variability in the sequences of actions and activities (as indicated by symbols in the legend).

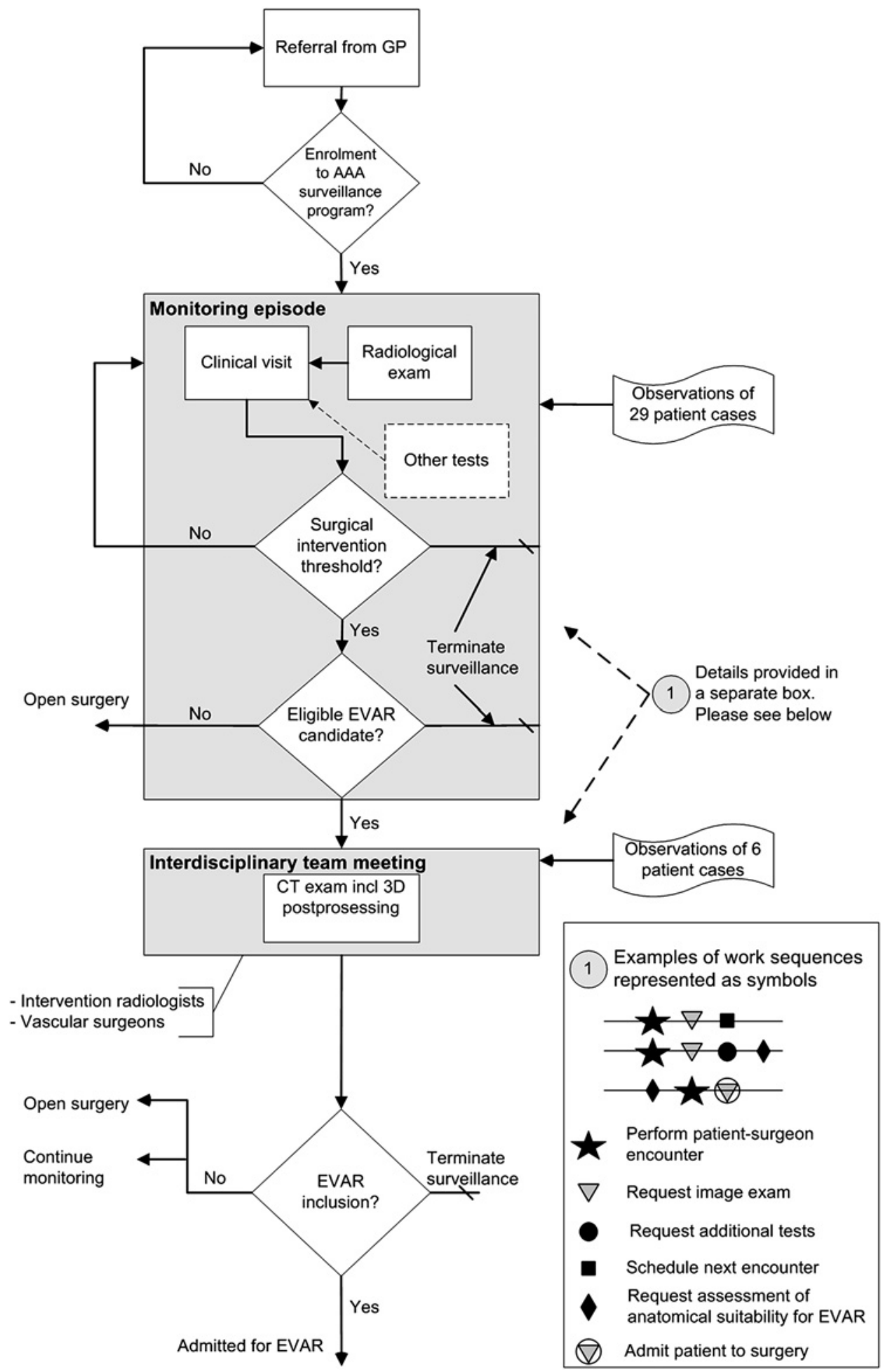

The observed process variations could be categorised as to whether the participating actors had planned for the variations or not. When the process was executed, planned variations were expected to occur. In contrast, unplanned variations were not expected to occur. Table 1 gives examples of these two kinds of variations. Table 1 also classifies variations according to what caused the variations. Some process variations could be attributed to the patient-for example, patient preferences, pathology, anatomy of the aorta, comorbidities or the patient's overall life expectancy (see figure 2). Suboptimal availability and/or use of resources also led to variation, meaning that planning of each episode of monitoring had to account for delays due to waiting lists and result turnaround time at radiological and other laboratory services. A third source of variation was suboptimal availability and/or use of EVAR resourcesfor example, process turnaround time related to the 
Overall description:

- Patients diagnosed as having abdominal aortic aneurysm were referred by the GP to the hospital's vascular surgery service.

- Each episode of monitoring consisted of at least two interdependent activities: (1) a radiological exam and (2) a surgeon-patient encounter at the outpatient clinic. Some patients also required additional activities such as heart-and-lung tests.

- At the community hospitals, the radiological/additional test activities were mainly performed in-house. One hospital scheduled the ultrasound exams to be performed on the same day as the clinical visit. The other hospital scheduled the radiological exams to be performed in timely advance of the scheduled clinical visit, taking into account the radiological report turnaround time.

- At the university hospital, both in-house and outside units performed image services and additional tests, impacting the report turnaround time.

Surgeon-patient encounter (clinical visit):

- A nurse prepared prerequisite information in advance such as GP referral for new patients, the record note from the previous monitoring episode, image and test reports.

- The nurse guided patients to the examination room, calming down anxious patients and providing information about the examination if necessary.

- The surgeon (a) studied the record note/GP referral, the image report or on-screen presentation of the image exam and possible test results; (b) conversed with the patient about issues such as aneurysm-related parameters, patient-related factors such as comorbidities and/or preferences, advice about self-care and future healthcare acts; (c) dictated a medical note stating the event and decision, including the patient's forthcoming care plan; and (d) requested imaging exams/tests and scheduled the next episode of monitoring.

When the patient's condition reached surgical intervention threshold:

- The surgeon referred eligible endovascular repair (EVAR) candidates to a preoperative anatomical suitability assessment. The decision pro or con EVAR took place during an interdisciplinary team meeting at the radiological department (university hospital). Both vascular surgeons and intervention radiologists participated in the team meeting. The local surgeon directed eligible EVAR patients to the vascular surgery service of the university hospital. Patient preferences influenced the decision on treatment options.

EVAR service. Variations that were anticipated (planned) factored in these potential causes. Variation that developed unexpectedly could be classified as: (a) failure of the actor to execute a planned task (ie, errors in execution $)^{24}$-for example, when a radiological exam had not taken place; or (b) the actor executing a task other than what had been planned for (ie, errors in planning), ${ }^{24}$-for example, when a surgeon reversed the decision to perform surgery on a patient that had been scheduled for operation.

For all actors, the safety and quality of patient care were major issues. All surgeons explained in detail how assessment and mitigation of risks were an integral part of the AAA surveillance programme. One reason for this was their concern about the complex trade-offs and uncertainties in decision-making. They typically linked the risk to specific 'worst case scenarios,' such as rupture of AAA in patients excluded from surgery, or patients accidentally dropping out of the surveillance loop. Surgeons at all units upheld a low number of ruptured AAA as a major criterion for their success, as exemplified in this statement from an interview:

...when we look at the number of ruptured [aneurysms], it appears that we perform better than world average. Maybe [our] mortality rate is about $30-40 \%$ versus $50 \%$ [in other countries]. However, we do not know this for sure... (Surgeon 1, university hospital)
The actors applied different strategies to minimise the number of ruptures and patient drop-outs. Some surgeons left nothing to the GPs, and included nearly all referred patients with an aneurysm into the screening programme. Other surgeons left patients having a stable aneurysm to be monitored by the GP until it reached a certain diameter $(4-4.5 \mathrm{~cm})$. Another strategy was to contact and reappoint patients who failed to show up for a consultation.

The broadened criteria for inclusion of patients to EVAR contributed to variations in the choice of treatment method, largely due to a lack of data about the long-term outcomes of the procedure. In particular, surgeons from community hospitals expressed unease about scheduling younger AAA patients to assessment for EVAR suitability. One surgeon from the university hospital said that:

The lower age limit for EVAR inclusion is $60 \ldots$ If this EVAR technology turns out to be stabilized for some time, this age limit may be lowered. (Surgeon 1, university hospital)

In contrast, a surgeon from one of the community hospitals explained that:

....we do not take a clear stand on this issue. Probably we disagree ourselves, and maybe we should not [be united], either. [In this part of the health region] I believe we practice a lower age limit of approximately 70-at least 


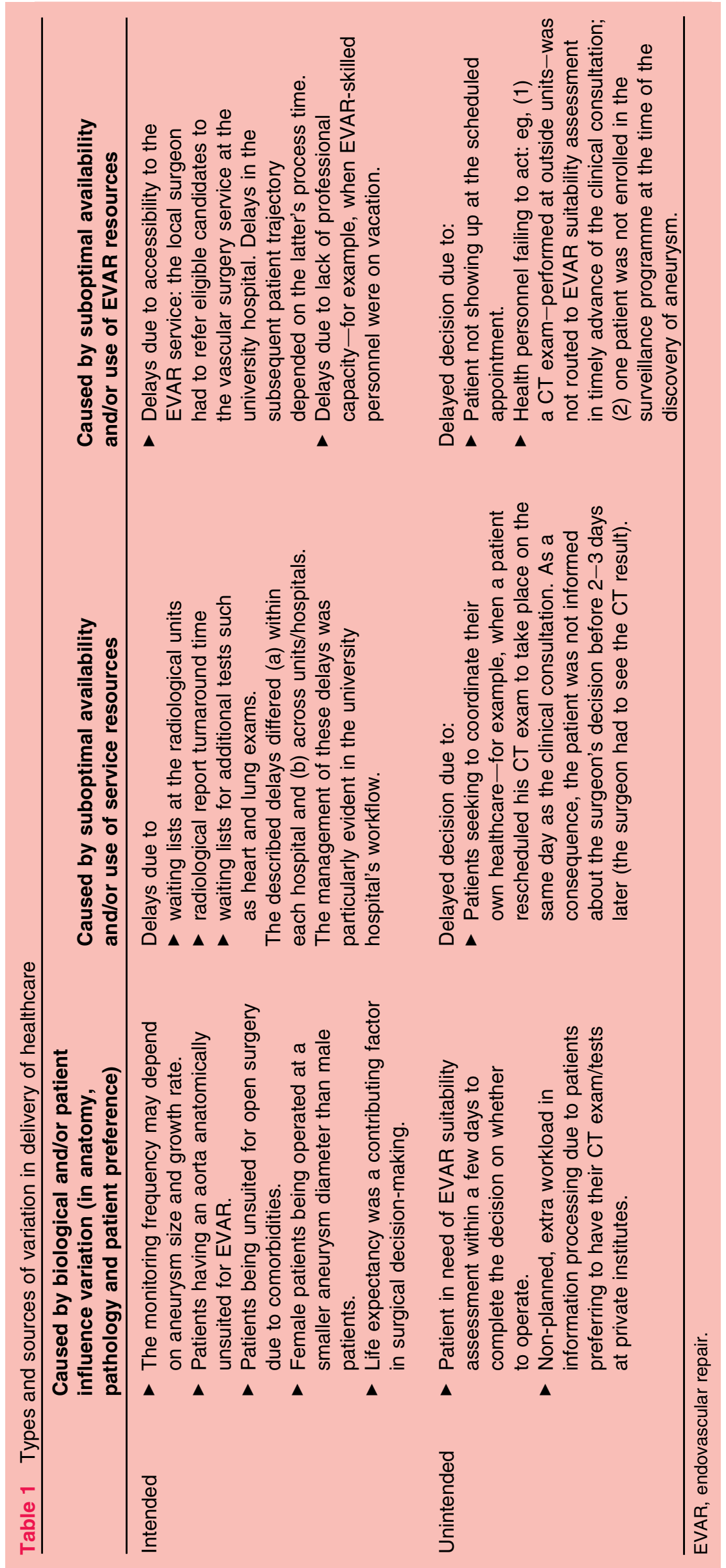



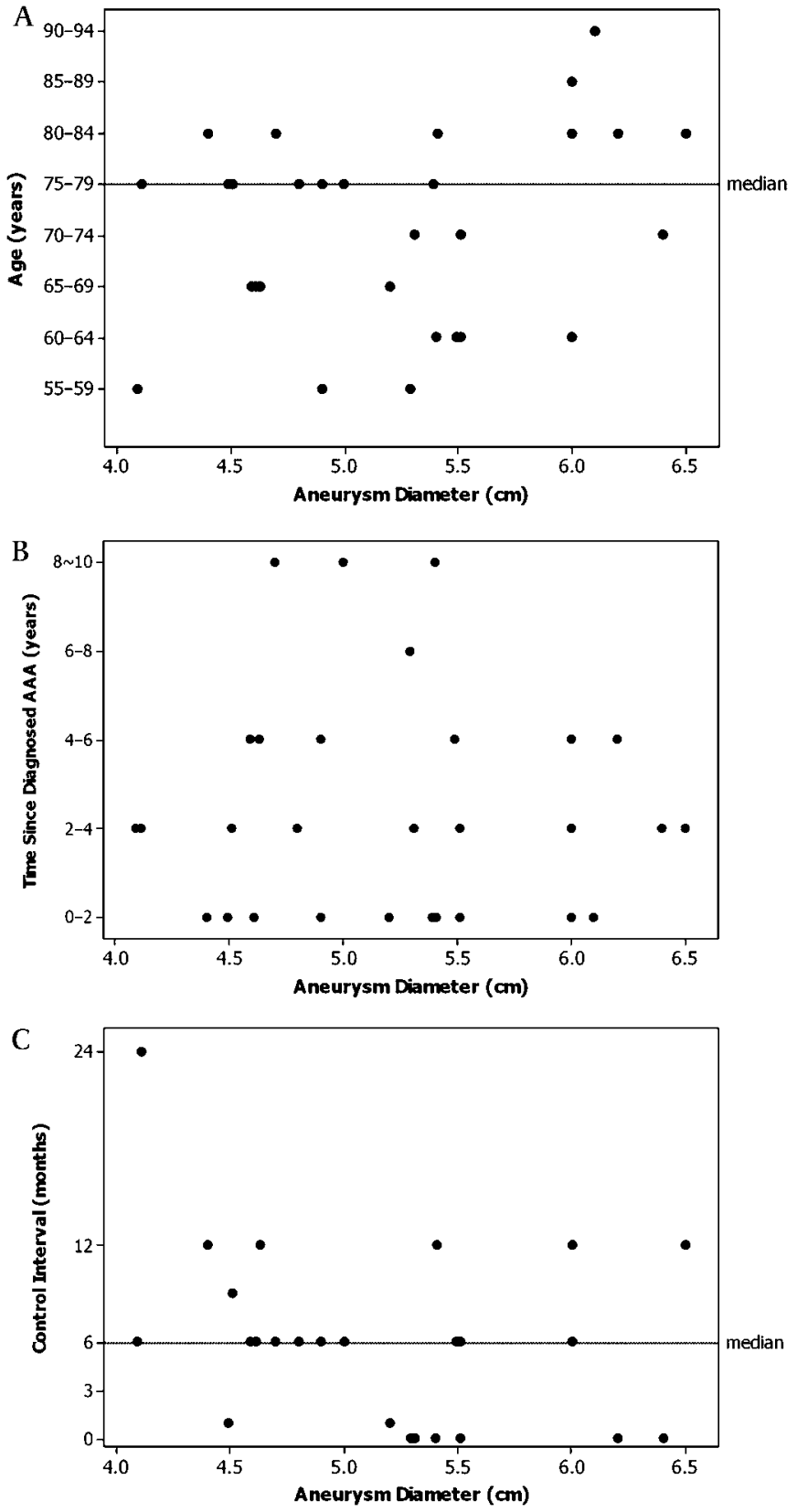

Figure 2 (A) Scatter plot of age against aneurysm diameter. (B) Scatter plot illustrating the time since first diagnosis of abdominal aortic aneurysm (patient-reported) against aneurysm diameter. (C) Scatter plot illustrating the time to the next episode of monitoring against aneurysm diameter. Code value ' 0 ' means that the patient was scheduled for operation. (The plot excludes two patients terminating the abdominal aortic aneurysm surveillance programme, and two patients without available data).

this accounts for my practice. [...] yes-provided that there are no contraindications [against the open surgery option]. (Surgeon 5, local hospital)

The difference in practice behaviour between 'actors who were' and 'actors who were not' involved in EVAR reflected the challenge of convincing local actors to have trust in the procedure and to account for developments of new EVAR technologies in risk assessments and decision-making.

\section{DISCUSSION}

In this study, we have reported on the dimensions, types and causes of variations in the monitoring of patients with aortic aneurysm, a clinical process that unfolds itself in collaboration between multiple institutions and departments. Our approach was designed to provide a detailed understanding of the characteristics of process variability, and with the purpose of elucidating requirements for an IT-system.

We found that the care process for AAA patients was best described as systematic analysis and mitigation of risks. Much of the observed variation was intended. Such intended 'natural' variety of protocols observed should not be considered a systematic threat for patient safety, but rather as a necessary risk mitigation activity. Activities were planned while taking into account the need for flexibility and opportunity to make last-minute adjustments, given these were required to achieve particular clinical aims and/or to accommodate to the preferences of the patient. However, risk analysis extended beyond that of traditional clinical problem-solving and decisionmaking. Other risks were related to the reliability of the healthcare system. For instance, having decided that a patient should be operated within, for example, 6 weeks, the surgeon could not blindly trust that the scheduling department actually would schedule the patient for surgery within this time limit. Existing IT systems had little support for mechanisms specifically tailored to scheduling and knowledge building. Rather than being built into the schema of the healthcare system, these mechanisms were reflected as characteristics of the actors' actions. To mitigate risks associated with omission or postponement of planned activities, clinicians sometimes added extra loops to the workflow such as an extra ultrasound examination or another consultation. This behaviour, which can be described as resilience behaviour, compensated for the lack of more formal mechanisms of resilience in the healthcare system, enhancing patient safety.

In our opinion, these findings should have a major influence on the design of IT systems to be implemented in this domain. Despite that, medical technology now pervades healthcare systems; the provision of care to the patient remains a human endeavour, strongly influenced by the experiences, skills and knowledge of the healthcare professional. We propose that IT-based process support for clinical work should be designed to enhance two essential capabilities of a resilient healthcare system. The first involves the capability of awareness, which refers to a mental state of the actors that result from 
them having available information about the actual execution and status of ongoing processes. These might be related to individual patients as well as to the cohort of patients under surveillance. We believe that enhanced awareness resulting from such IT support could prevent process breakdowns, reducing the risk of patient dropouts and other safety threats.

The second is the capability to gain knowledge from experience. This capability refers to the healthcare system's ability to enhance its knowledge and support a continuous learning process among involved actors based on data about its processes and outcomes. Support for this capability is particularly important for continually evolving trans-institutional care processes that involve more than one treatment alternative. Such support could alleviate the challenge of individual development of new surgical skills while also maintaining existing skills in standard treatment, provide effective dissemination of data to substantiate the new procedure's effectiveness and reduce competencyrelated risks for patients. ${ }^{25}{ }^{26}$ In order to support the generation and dissemination of knowledge, the system should provide the following:

1. Feedback on process and outcome parameters for the patient group to all clinicians involved. This feedback should include the same information on patients who experience aneurysm rupture in order to learn from possible 'false negative' cases (=patients wrongly excluded from surgery). This is important because the mechanisms that lead to aneurysm rupture are still largely unknown.

2. Real-time process information to the local practitioners, alleviating potential practice uncertainties for clinicians not directly involved in the EVAR activity with respect to both the individual patient at hand and the patient group at the related meso-level.

Providing IT support for the capabilities of awareness and that of gaining knowledge from experience will enhance actors' risk-mitigating ability by strengthening Hollnagel's cornerstones of resilience. ${ }^{17}$ Moreover, our suggestions on IT design are in line with Hollnagel's proposition of resilience as a result of a dynamic process in which qualities linked to the four cornerstones have to be exercised continuously. ${ }^{14}$ The outlined IT support could very well also lead to more intended process variations, while limiting unintended process variations. Similar to our findings, studies of the effects of computerised clinical guidelines and decision-support systems find that when clinicians indicate that their actions are exceptions, these deviations are often well justified. ${ }^{27-29}$ As mentioned in the introduction, modern theory on quality emphasises the importance of reducing variation. We suggest that efforts to reduce variation should focus on unintended process variation that cannot be attributed to risk mitigation in clinical problem-solving. Unfortunately, distinguishing between unintended and intended process variation is not necessarily a straightforward task. Further complicating work to improve quality by reducing variability in highly complex healthcare settings, Ashby's requisite law states that there must be a possible response for every type/source of variation impacting upon a system. ${ }^{30} 31$ There is, however, important common ground between quality improvement and resilience theory. For instance, both theories view continuous learning from exceptions as a source for improving safety, and place emphasise on structures, procedures and rules. Distinguishing between the two, resilience thinking also stresses the importance of human performance in dealing with continually changing work practice, and also draws heavily on opportunities for learning from successes.

While enhancing resilience with IT support seems necessary, introducing such measures may generate new challenges. If multiple types of variations take place at the same time, the cumulative effect might create situations that increase the risk, or even contribute to major failure, denoted by Hollnagel as 'functional resonance. ${ }^{32}$ As for the AAA care process, consequences of such resonances may have negative implications for the assessment of acceptable rates of patient drop-outs and aneurysm ruptures. Moreover, all involved active actors may contribute to resilience. Given a definition of the boundaries of the AAA surveillance and treatment process, intended variations may be caused by factors inside the care process itself (endogenous) or by factors in the environment/context (exogenous). Because of the somewhat surgeon-centric perspective of our study, we cannot generally rule out that other healthcare actors (eg, the EVAR radiologists and the primary-care physicians of the patient) also contributed to resilience as well as to the risk of functional resonance. Interactions with and effects from the surrounding environment and actors need to be taken into account when tailoring IT support, that is to enhance resilience to a given framework of functions aiming to enhance patient safety.

To summarise, IT-based process solutions should be designed to facilitate clinicians' resilience performance when adapting to an evolving care process. With respect to the cross-organisational AAA care, such systems should support risk-mitigating decision-making and prevent patients falling out of the surveillance loop. Our case is a limited sample, drawn from a very small part of the healthcare system. This limits the generalisability of our findings. Nevertheless, our approach should provide important insights for the design of process support systems for our case that may also have broader applicability. 
Acknowledgements We wish to thank the participants from the three case hospitals who were willing to participate in the study. We would also like to thank PhD candidate A Landmark, for technical help with the manuscript.

Funding BB was funded by Sør-Trøndelag University College, and AS by the Research Council of Norway (VERDIKT).

Competing interests None.

Ethics approval Ethics approval was provided by the Regional Committee for Medical Research Ethics and the Norwegian Social Science data Services.

Provenance and peer review Not commissioned; externally peer reviewed.

\section{REFERENCES}

1. Chaudhry B, Wang J, Wu S, et al. Systematic review: impact of health information technology on quality, efficiency, and costs of medical care. Ann Intern Med 2006;144:742-52.

2. Greenhalgh T, Potts HW, Wong G, et al. Tensions and paradoxes in electronic patient record research: a systematic literature review using the meta-narrative method. Milbank $Q$ 2009;87:729-88.

3. Panzarasa S, Quaglini S, Cavallini A, et al. Computerised guidelines implementation: obtaining feedback for revision of guidelines, clinical data model and data flow. Artif Intell Med 2007;4594:461-6.

4. Stefanelli M. Knowledge and process management in health care organizations. Methods Inf Med 2004;43:525-35.

5. Montgomery DC. Introduction to Statistical Quality Control. Hoboken, NJ: Wiley, 2005.

6. Olatunde AA. On the importance of statistical process control in health care. Res J Med Sci 2009;3:87-90.

7. Peek N, Goud R, Abu-Hanna A. Application of statistical process control methods to monitor guideline adherence: a case study. AMIA Annu Symp Proc 2008:581-5.

8. Thor J, Lundberg J, Ask J, et al. Application of statistical process control in healthcare improvement: systematic review. Qual Saf Health Care 2007;16:387-99.

9. Deming WE. Out of the Crisis. Cambridge, MA: MIT Press, 2000

10. Pentland BT. Sequential variety in work processes. Organ Sci 2003;14:528-40.

11. Berwick DM. Controlling variation in health care: a consultation from Walter Shewhart. Med Care 1991;29:1212-25.

12. Thompson AG. Variation may be good for your health: the nature of evidence. Int J Qual Health Care 2002;14:1-3.

13. Costella MF, Saurin TA, de Macedo Guimarães LB. A method for assessing health and safety management systems from the resilience engineering perspective. Saf Sci 2009;47:1056-67.

14. Hollnagel E, Woods DD, Leveson N, eds. Resilience Engineering Concepts and Precepts. Aldershot, UK: Ashgate Publishing Limited, 2008.
15. Nemeth CP, Hollnagel E, Dekker S, eds. Preparation and Restoration. Farnham, UK: Ashgate Publishing Limited, 2009.

16. Jeffcott SA, Ibrahim JE, Cameron PA. Resilience in healthcare and clinical handover. Qual Saf Health Care 2009;18:256-60.

17. Hollnagel $\mathrm{E}$. The four cornerstones of resilience engineering. In: Nemeth CP, Hollnagel E, Dekker S, eds. Preparation and Restoration. Farnham, UK: Ashgate Publishing Limited 2009:117-33.

18. Tan WA, Makaroun MS. Abdominal aortic aneurysm, rupture. http:// emedicine.medscape.com/article/416397-overview (accessed 4 Feb 2009).

19. Fillinger $M$. Who should we operate on and how do we decide: predicting rupture and survival in patients with aortic aneurysm. Semin Vasc Surg 2007;20:121-7.

20. Wilt $\mathrm{T}$, Lederle $\mathrm{F}$, Macdonald R, et al. Comparison of endovascular and open surgical repairs for abdominal aortic aneurysm. Evid Rep Technol Assess (Full Rep) 2006;144:1-113.

21. Chambers D, Epstein D, Walker S, et al. Endovascular stents for abdominal aortic aneurysms: a systematic review and economic model. Health Technol Assess 2009;13:1-318.

22. Brattheim B, Seim AR, Faxvaag A. Clinical processes in an innovative vascular surgeon community. Implications for workflow modeling. Stud Health Technol Inform 2008;136:371-6.

23. Sørby ID, Nytrø Ø, Røst TB. Empirical Grounding of Guideline Implementation in Cooperative Clinical Care Situations. Riva del Garda, Italy: Al Techniques in Healthcare: Computerized Guidelines and Protocols, 2006.

24. Kohn LT, Corrigan J, Donaldson MS, eds. To Err Is Human: Building a Safer Health System. Washington, DC: National Academy Press, 2000.

25. Johnson J, Rogers W, Lotz M, et al. Ethical challenges of innovative surgery: a response to the IDEAL recommendations. Lancet 2010;376:1113-15.

26. Angelos $P$. The ethical challenges of surgical innovation for patient care. Lancet 2010;376:1046-7.

27. Garg AX, Adhikari NK, McDonald H, et al. Effects of computerized clinical decision support systems on practitioner performance and patient outcomes: a systematic review. JAMA 2005;293:1223-38.

28. Quaglini S, Stefanelli M, Cavallini A, et al. Guideline-based careflow systems. Artif Intell Med 2000;20:5-22.

29. Persell SD, Dolan NC, Friesema EM, et al. Frequency of inappropriate medical exceptions to quality measures. Ann Intern Med 2010;152:225-31.

30. Nemeth CP, O'Connor M, Cook RI. The infusion device as a source of healthcare resilience. In: Nemeth CP, Hollnagel E, Dekker S, eds. Preparation and Restoration. Farnham: Ashgate Publishing Limited, 2009:235-53.

31. Ashby WR. An Introduction to Cybernetics. London: Chapman \& Hall, 1956. Internet (1999). http://pcp.vub.ac.be/books/IntroCyb.pdf (accessed 10 Dec 2010).

32. Hollnagel E. Barriers and Accident Prevention. Aldershot, UK: Ashgate Publishing Limited, 2004. 